\title{
Miniaturized analysis of amylopectin chain length distribution by Fluorescence-assisted Capillary Electrophoresis (FACE) down to single starch granules
}

Amandine Pruvost ${ }^{1}$, Stanislas Helle ${ }^{1,2,3}$, Nicolas Szydlowski ${ }^{2}$, Christian Rolando $*, 1,4$

${ }^{1}$ Univ. Lille, CNRS, USR 3290 - MSAP - Miniaturisation pour la Synthèse, 1'Analyse et la Protéomique, Lille, France

${ }^{2}$ Univ. Lille, CNRS, UMR8576 - UGSF - Unité de Glycobiologie Structurale et Fonctionnelle, Lille, France

${ }^{3}$ Florimond Desprez Veuve-et-Fils Ind., Section Biotechnologies, BP 41, 59242 Cappelle en Pévèle, France

${ }^{4}$ Shrieking Sixties, 1-3 Allée Lavoisier, 59650 Villeneuve-d'Ascq, France

* Correspondence: christian.rolando@univ-lille.fr; Tel+33-320434977

\begin{abstract}
In the present work, we developed a miniaturized method for determining amylopectin chain length distribution (CLD) by fluorescence-assisted capillary electrophoresis (FACE). The method relies on single granule entrapping into capillaries followed by direct starch gelatinization and amylopectin debranching on carbographbased solid phase extraction (SPE) cartridges. Sample desalting on Hypersep ${ }^{\mathrm{TM}}$ tips following APTS-labelling and the use of nanovials allowed for the fluorescence analysis of weakly diluted samples. Consequently, method sensitivity was improved by 500 -fold which is compatible with the analysis of single potato starch granules. The method was implemented to determine CLD profiles of single starch granules ranging from 50 to $100 \mu \mathrm{m}$ in diameter. In these experiments, the relative proportion of starch glucans of up to 30 degrees of polymerization (DP) could be quantified.
\end{abstract}




\section{INTRODUCTION}

Starch is naturally present in plants and is composed of glucose residues linked together by $\alpha-1.4$ and branched by $\alpha-1.6$ O-glycosidic bonds (1). The latter form two characteristic polymers, amylose and amylopectin. Amylopectin is a moderately branched, highly ordered molecule conferring crystallinity to starch granules (2). The enzymatic machinery responsible for amylopectin synthesis have been studied extensively (3). However, starch granule initiation as well as glucan assembly and organization are not yet fully understood (4). Many methods were implemented to characterize starch polysaccharides and granule structure or morphology (5). Apart from microscopic methods (eg., electron microcopy, atomic force microscopy), microbeam X-ray-based methods (ie., diffraction and microfluorescence) and second harmonic generation that allow characterizing single granules, analytical methods require the use of relatively large samples containing at least thousands of granules originating from whole organs or organisms (5-7). In the latter cases, observed averages may hide discrete events that could reflect biological phenomena potentially important for our understanding of starch metabolism.

Important structural information can be provided by chain length distribution (CLD) analysis (8). Determining the degree of polymerization (DP) of glucose chains requires the foregoing enzymatic release of branched glucans with the use of debranching enzymes (isoamylase and pullulanase) (5). Both size exclusion chromatography and field flow fractionation have been used to separate glucans as well as native, branched amylopectin molecules ( 9 , 10). On the other hand, mass spectrometry, especially MALDI-TOF using the matrix 2,5-Dihydroxybenzoic acid (DHB), was successfully applied to determine CLD of native and chemically modified starches after enzymatic debranching (11). However, accurate quantification of glucan families is difficult because of the desorption heterogeneity between glucans according to their size. The drawbacks of both MALDI-TOF and LC-ESI-MS mostly rely on the difficulty for ionizing glucans above DP 30. The most efficient methods for determining CLD of native starches are high performance anion exchange chromatography with pulsed amperometric detection (HPAEC-PAD) and fluorescence-assisted capillary electrophoresis (FACE) (5). Although requiring chemical derivatization (usually by reductive amination with 8-aminopyrene-1,3,6-trisulfonic acid, APTS) of the oligosaccharides, FACE is highly sensitive compared to HPAEC-PAD and allows for accurate quantification of the longest glucans. The sensitivity threshold of the fluorescent detection of APTS-derivatized sugars was established at the fg scale (12). However, method sensitivity is limited by sample preparation and the minimum volume necessary for hydrodynamic CE injection.

In the present study, we developed an optimized protocol to avoid dilution and the loss of material during sample preparation. Amylopectin debranching was directly performed on carbograph-based solid phase extraction (SPE) cartridges. Sample desalting on hypersep tips following derivatization and the use of nanovials allowed for the analysis of non-diluted $5 \mu \mathrm{L}$ samples. This protocol improved method sensitivity by 500 -fold and allowed to determine CLD profiles of single potato starch granules ranging from 50 to $100 \mu \mathrm{m}$ in diameter. 


\section{EXPERIMENTAL SECTION}

Materials and chemicals. All solutions were prepared using ultrapure water purified with a MilliQTM Academic system (Merck Millipore, Burlington, Massachusetts, United States). Extract CleanTM SPE Carbo (150 mg/4 mL) were purchased from Grace Compagny. Hypercarb®. APTS (8-aminopyrene-1,3,6-trisulfonic acid trisodium salt), $1 \mathrm{M}$ sodium cyanoborohydride in THF, Isoamylase, pullulanase and all solvents were purchased from Sigma Aldrich (Saint-Louis, Missouri, USA).

Starch isolation. Starch was extracted from tubers of Solanum tuberosum (cv. Monalisa) cultivated in soil in Villeneuve d'Ascq (50.607735, 3.143431), France, between March and July 2014. Tubers (approximately 70 g) were cleaned with tap water and peeled before the extraction. Tubers were crushed with a blender in $200 \mathrm{~mL}$ of milli-Q water. The extracts were then filtered with a nylon net (100 $\mu \mathrm{m}$ mesh width) and left during $3 \mathrm{~h}$ for starch granule sedimentation. The supernatant was then discarded and the starch pellet was resuspended in $500 \mathrm{~mL}$ of milli-Q water. The starch suspension was subsequently washed 3 times with $1 \mathrm{~L}$ of milli-Q water and stored in $20 \%$ ethanol at $4{ }^{\circ} \mathrm{C}$.

Capture of single starch granules. The starch suspension was diluted 50 times, placed on a glass slide, and single starch granules were captured by capillarity within uncoated silica columns (360 $\mu \mathrm{m}$ outer diameter, $100 \mu \mathrm{m}$ inner diameter, $2 \mathrm{~cm}$ length). The presence of starch granules within capillaries was confirmed with the use of an optical microscope (Optika, Italy).

Amylopectin debranching and Extract Clean ${ }^{\mathrm{TM}}$ desalting. Starch was heated at $100{ }^{\circ} \mathrm{C}$ during 15 minutes with $125 \mu \mathrm{L}$ of miliQ water in a preequilibrated (acetonitrile (ACN) / water; v/v) Extract CleanTM Carbograph cartridge. Then, $125 \mu \mathrm{L}$ of $55 \mathrm{mM}$ sodium acetate, $\mathrm{pH} 3.5$ and varying amounts of isoamylase and pullulanase were added prior to overnight incubation at $42{ }^{\circ} \mathrm{C}$. Enzyme quantities were adjusted according to the starting amount of starch with ratios of $10 \mathrm{U} / \mathrm{mg}$ of starch and $0.9 \mathrm{U} / \mathrm{mg}$ of starch for isoamylase and pullulanase, respectively. After debranching, the flowthrough was discarded with a vacuum pump. The cartridge was then washed once with $2 \mathrm{~mL}$ of water and glucan elution was performed with $500 \mu \mathrm{L}$ of acetonitrile $(\mathrm{ACN}) /$ water $(50: 50$; v/v).

APTS-derivatization and hypersep ${ }^{\mathrm{TM}}$ desalting. After elution, samples were dried in a SpeedVac ${ }^{\mathrm{TM}}$ Concentrator (EppendorfTM Concentrator Plus, Eppendorf, Hamburg, Germany) at $45{ }^{\circ} \mathrm{C}$ until total evaporation. Then, $2 \mu \mathrm{L}$ of $200 \mathrm{mM}$ 8-aminopyrene-1,3,6-trisulfonic acid trisodium salt (APTS) in 15\% acetic acid and $2 \mu \mathrm{L}$ of $1 \mathrm{M}$ sodium cyanoborohydride in tetrahydrofuran (THF) were added prior to overnight incubation at $42{ }^{\circ} \mathrm{C}$ in darkness. $46 \mu \mathrm{L}$ of water were added to obtain a final volume of $50 \mu \mathrm{L}$. Excess APTS and salts were removed on a Hypersep® tip $(10-200 \mu \mathrm{L})$. The tip was washed with $50 \mu \mathrm{L}$ of $100 \%$ ACN and equilibrated with water by performing 20 suction/discharge steps. Then, the sample was loaded on the stationary phase by performing 30 suctions/discharges. Washes were carried out with 20 suctions/discharges in $50 \mu \mathrm{L}$ of washing solution $(0.1 \%$ formic acid (FA) in water). Glucans were eluted in two steps using 50:50 ACN: 0.1\% FA and 100\% ACN with 0.1\% FA. Glucan 
samples were dried in a SpeedVac ${ }^{\mathrm{TM}}$ Concentrator (EppendorfTM Concentrator Plus, Eppendorf, Hamburg, Germany) at $45{ }^{\circ} \mathrm{C}$ until total evaporation.

Capillary electrophoresis analysis. Glucans were resuspended in ultrapure water for capillary electrophoresis. For the analysis of samples above $50 \mu \mathrm{g}$, labelled polysaccharides were resuspended in $50 \mu \mathrm{L}$ of water and subsequently diluted 5 times. For the study of samples below $50 \mu \mathrm{g}$ as well as single granules, labelled glucans were resuspended in $5 \mu \mathrm{L}$ of water and directly used for injection with the use of nanovials (Sciex Separations, Les Ulis, France). Electrophoresis was performed on reverse polarity with a Beckman Coulter PA 800 plus instrument (Sciex Separations, Les Ulis, France) equipped with a laser induced fluorescence (LIF) detector. Separation was performed in a $50 \mu \mathrm{m}$ i.d., $375 \mu \mathrm{m}$ o.d. bare fused silica capillary of $60.2 \mathrm{~cm}$ length (Sciex Separations, Les Ulis, France). Sample injection was carried out at 0.5 psi during $20 \mathrm{~s}$ and separation was achieved at $30 \mathrm{kV}$ in carbohydrate separation gel buffer (Sciex Separations, Les Ulis, France), diluted to 1/3 in ultrapure water. 


\section{RESULTS AND DISCUSSION}

FACE is based on the reductive amination of glucan reducing ends with APTS (scheme 1). This reaction leads to glucan labeling with a molar ratio of 1:1 allowing for accurate quantification of polysaccharides regardless of their molecular weight. Another advantage of the method relies on the sensitivity of fluorescent detection of APTS that was estimated at the fg scale (13). Therefore, this method is compatible with the characterization of limited sample amounts. However, high dilutions are necessary for good peak resolution and proper separation of the products following debranching and APTS-labeling. Although desalting is often performed after debranching, at least 50-fold dilutions are still used for proper peak shape and resolution following APTS-labeling. Salts and excess APTS are likely responsible for this phenomenon. Thus, even though the analysis of tiny molecule amounts is theoretically possible, routine sample preparation requires micrograms of starting material.
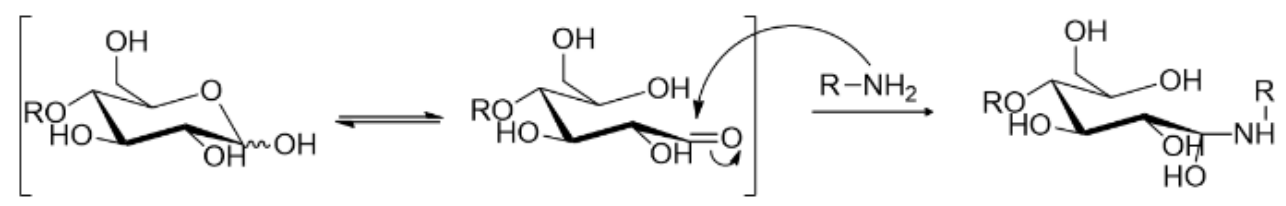

$$
\mathrm{H}_{2} \mathrm{O}-\|
$$

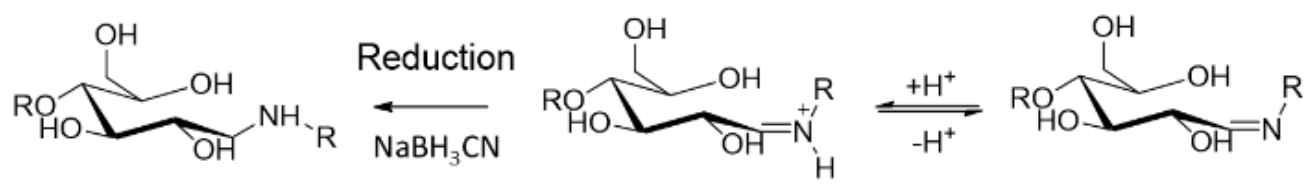

Scheme 1. Labeling reaction of glucan reducing ends by reductive amination using APTS.

\section{Impact of Extract Clean ${ }^{\mathrm{TM}}$ desalting on starch CLD}

We first investigated the impact of Extract CleanTM Carbograph based desalting of debranched glucans on the chain length distribution (CLD) of potato starch (Figure 1). $500 \mu \mathrm{g}$ of starch were submitted to enzymatic debranching and were either or not desalted on Extract CleanTM SPE cartridges before APTS-derivatization. The difference between both CLDs is shown in Figure 1.b and illustrates poor impact of this step on the relative abundance of each DP. 


\section{Effect of sample downsizing on starch CLD}

In order to analyze decreased amounts of starting material, an additional desalting step using HypersepTM tips was added between the derivatization and CE analysis steps. This allowed to remove salts and excess APTS and thus to directly inject non-diluted labeled samples. Moreover, nanovials were used to reduce sample volume down to $5 \mu \mathrm{L}$ for $\mathrm{CE}$ injection. Although hydrodynamic injection implies the analysis of only few nanoliters of sample, the minimum sample volume used with common vials is $50 \mu \mathrm{L}$. With these protocol adjustments, the weight of starting material could theoretically be decreased by 500 -fold.

Serial dilutions were performed following dispersion of starch polysaccharides by heat. $2 \mathrm{mg}, 500 \mu \mathrm{g}, 50 \mu \mathrm{g}, 5$ $\mu \mathrm{g}, 0.5 \mu \mathrm{g}$ or $0.05 \mu \mathrm{g}$ of starting material were then used for CLD analysis with the optimized protocol (Figure 2). Typical amylopectin CLDs display multimodal distributions varying according to plant species and genotypes. Starch from the potato cultivar Monalisa used in this study harbored a characteristic CLD profile corresponding to previous descriptions when $2 \mathrm{mg}, 500 \mu \mathrm{g}$ and $50 \mu \mathrm{g}$ of starting material were used (13) (Figure 2.a, 2.b and 2.c). However, the abundance of the longest DPs decreased with the amount of starting material. This indicates that DPs above 60 gradually dropped below the detection threshold of fluorescence detection. This effect was even more pronounced when $5,0.5$ or $0.05 \mu \mathrm{g}$ were used for the analysis with the clear disappearance of the second distribution mode corresponding to amylopectin B chains (14). Moreover, the differences between profiles of diluted samples and the non-diluted standard showed that the abundance of shorter glucans relatively increased with the decrease of longer DPs (Figure. 2). 
(a)

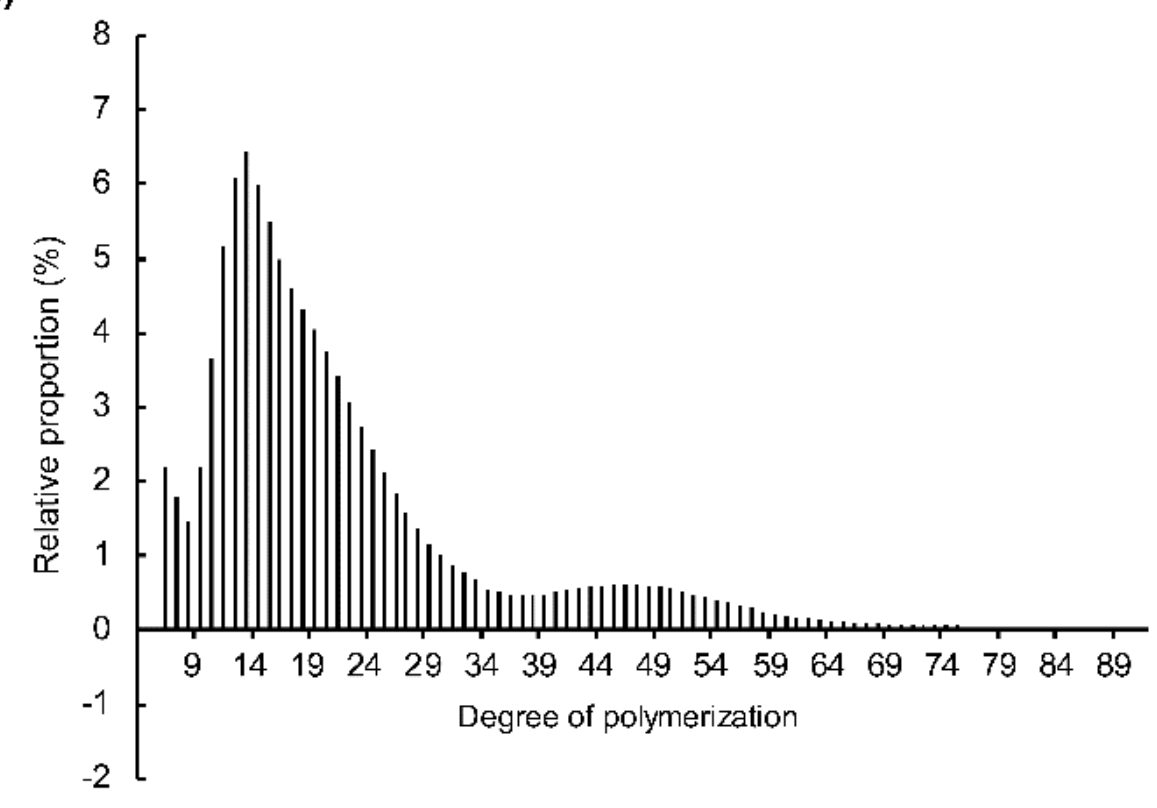

(b)

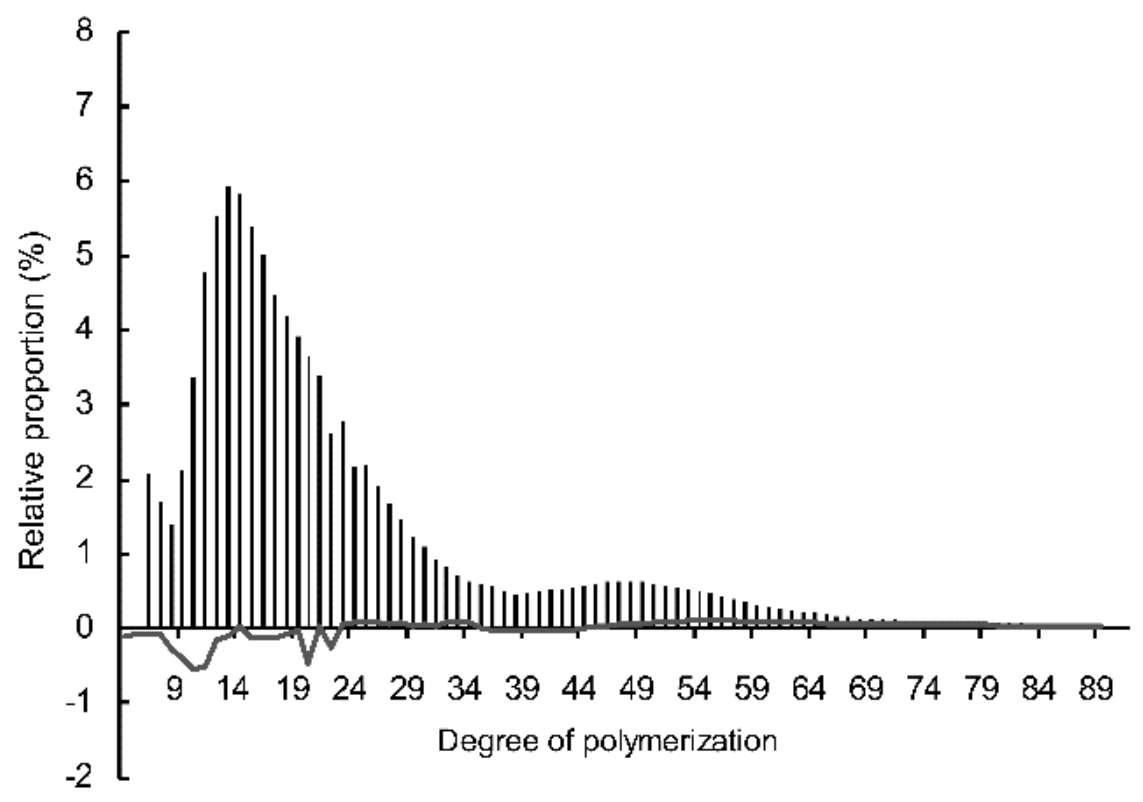

Figure 1. Impact of the Extract Clean ${ }^{\mathrm{TM}}$ desalting step on potato starch chain length distribution. (a) $500 \mu \mathrm{g}$ of potato starch were debranched with $5 \mathrm{U}$ of isoamylase and $0.45 \mathrm{U}$ of pullulanase prior to APTS-labeling and capillary electrophoresis analysis. The relative proportions of glucans ranging from a degree of polymerization (DP) of 6 to 90 were plotted. (b) same as in (a) with the addition of a desalting step using an Extract CleanTM carbograph cartridge prior to APTS-labelling. The line corresponds to the difference between profile (a) and (b). 
(a)

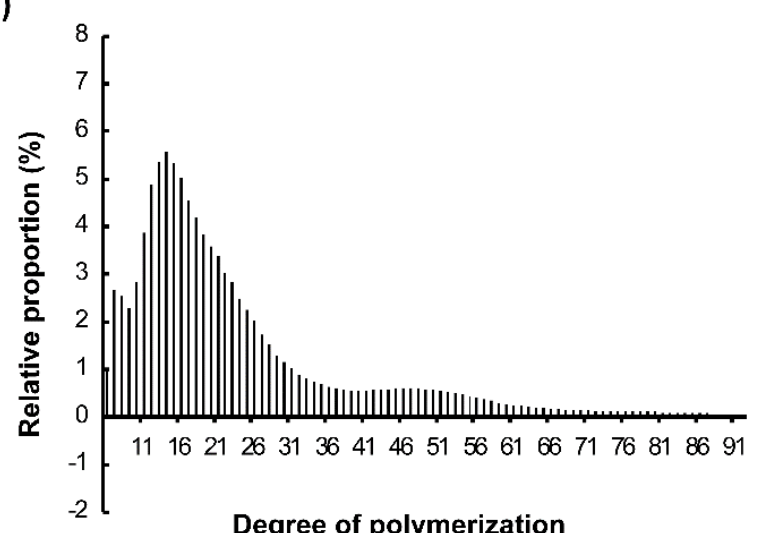

(d)

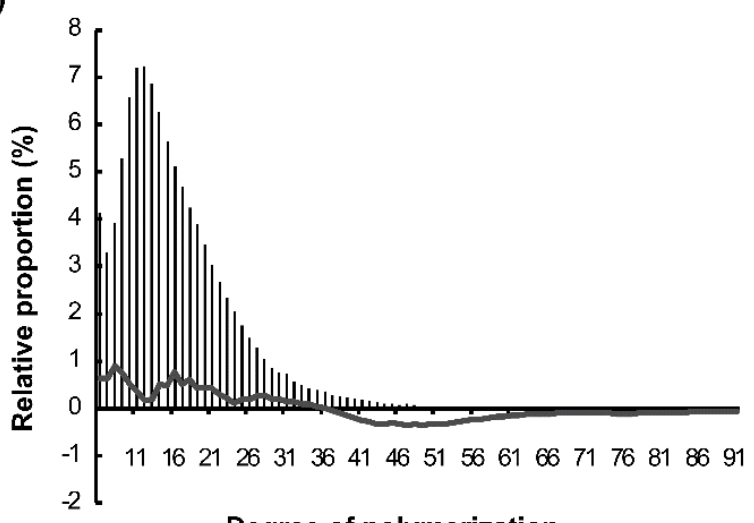

(b)

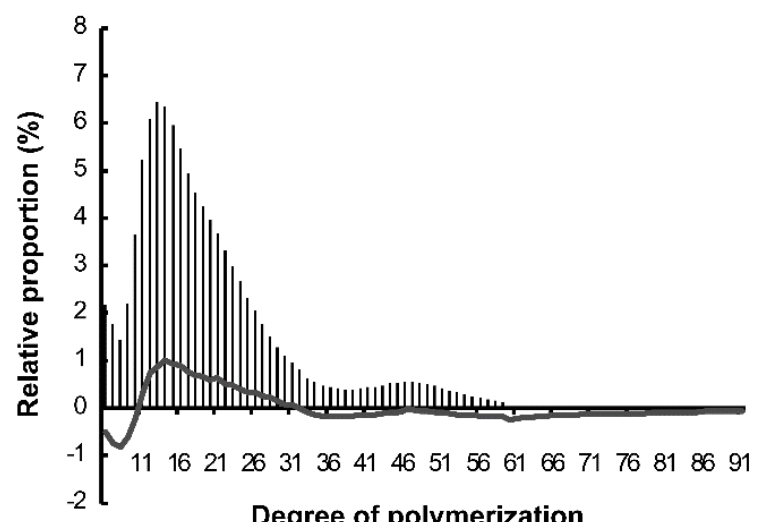

(e)

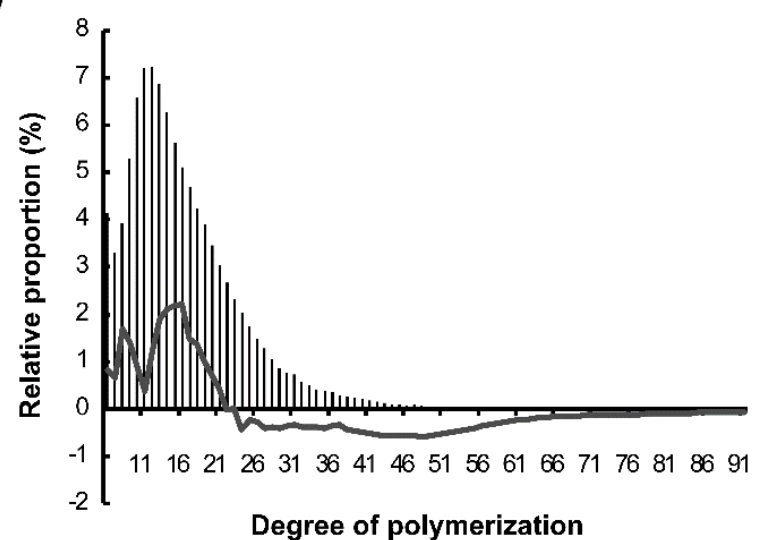

(c)

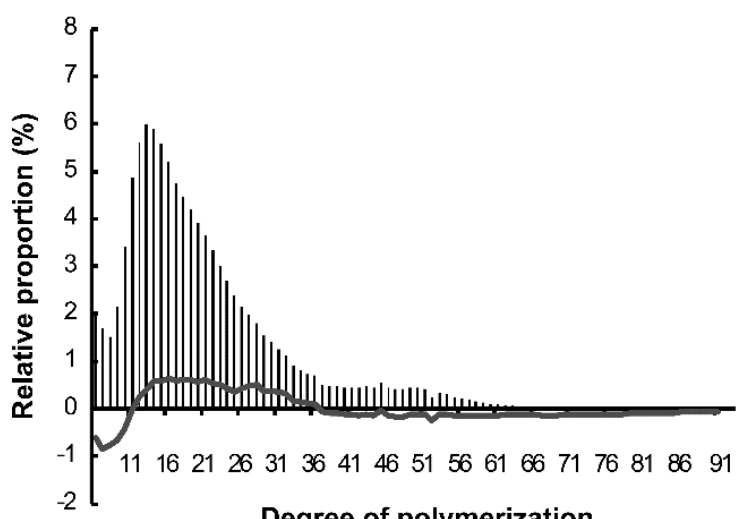

(f)

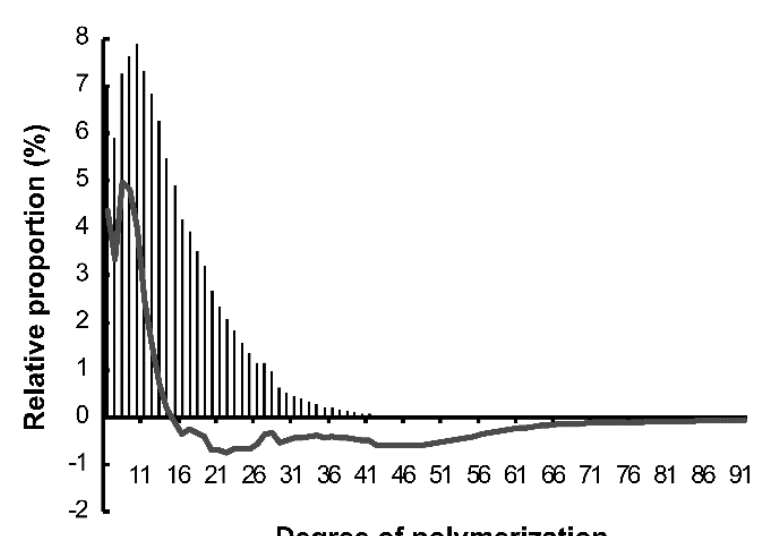

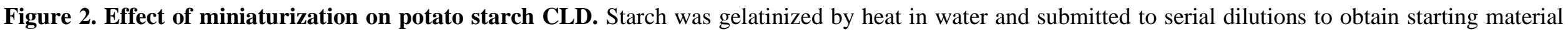

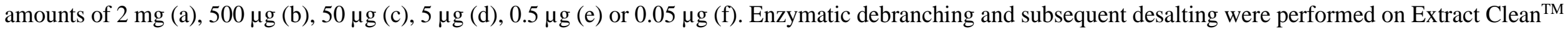

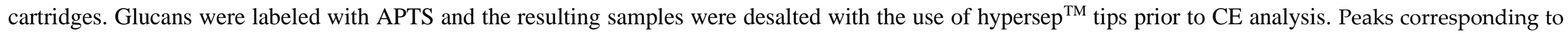

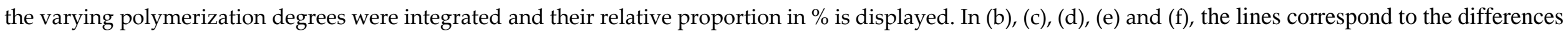
between the corresponding profiles and the reference profile presented in (a). 


\section{Single granule analysis}

Potato starch granules range between 4 and $100 \mu \mathrm{m}$ in diameter (13). We postulated that granules with diameters of about $50 \mu \mathrm{m}$ could provide enough material for single granule CLD analysis. Thus, a granule suspension was diluted prior to be placed onto a glass slide and capillary sections of $2 \mathrm{~cm}$ were used to catch individual granules by capillarity (Figure 3). The presence of one granule per section of capillary was confirmed by optical microscopy and granule size was estimated by image analysis. Individual granules were deposited directly into carbograph SPE cartridges for enzymatic debranching followed by the adapted sample preparation procedure and CE analysis (Figure 4).

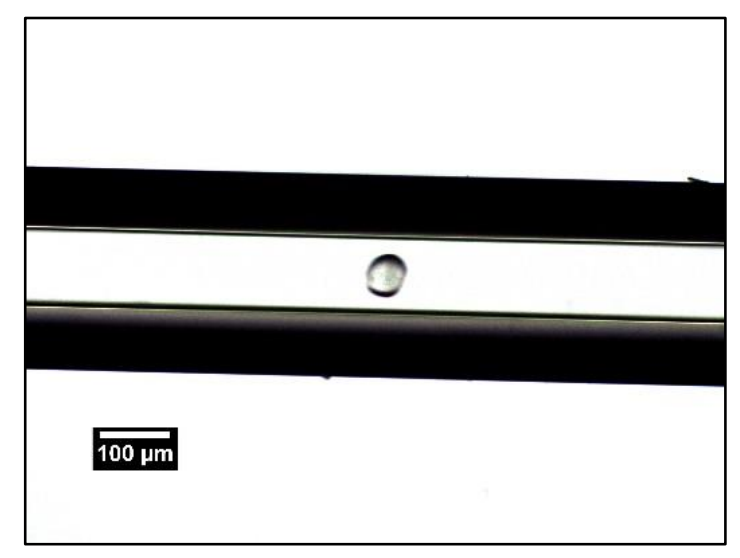

Figure 3. Starch granule entrapping into a silica capillary. A suspension of potato Monalisa starch granules was diluted and placed onto a glass slide prior to granule entrapping within a glass capillary $(360 \mu \mathrm{m}$ outer diameter, $100 \mu \mathrm{m}$ inner diameter, $2 \mathrm{~cm}$ length). Picture was taken with an optical microscope and granule diameter was estimated around $55 \mu \mathrm{m}$.

APTS-labeled glucans could be detected in the analysis of five individual granules ranging from $50 \mu \mathrm{m}$ to 70 $\mu \mathrm{m}$ in diameter (Figure 4.a). Although the fluorescence signal was near the sensitivity threshold, peaks could be integrated up to DP 30 (Figure 4.b). Among the analyzed granules, CLD profiles were highly similar, indicating that amylopectin structure is maintained between them. However, most amylopectin B-chains could not be detected. The development of labeling methods using fluorophores with higher response coefficients may help increasing method sensitivity and characterize longer amylopectin glucans from single potato starch granules or limited starch samples. 


\section{CONCLUSION}

Here, we report on the optimization of starch CLD analysis by FACE that was miniaturized to allow characterizing samples at the ng scale. Starch gelatinization, debranching and the first desalting step were performed in one pot on SPE cartridges. A second desalting step using hypersep ${ }^{\text {TM }}$ tips was added after APTS-labeling to remove salts and excess APTS thus permitting the analysis of undiluted samples. The latter step and the concomitant use of nanovials requiring $5 \mu \mathrm{L}$ minimal volume for injection increased method sensitivity by 500 -fold. The method was applied to the characterization of single potato starch granules and CLD could be determined up to DP 30 . This analysis showed little variations between five granules of comparable diameters. The main limitation of this optimized protocol is due to the relatively low abundance of glucans above DP 30 in starch, thus precluding the exhaustive analysis of chain length distribution. Further improvement of method sensitivity will require the use of fluorophore with better response coefficients. 


\section{REFERENCES}

1. Buleon A, Colonna P, Planchot V, Ball S. Starch granules: structure and biosynthesis. International Journal of Biological Macromolecules. 1998;23.

2. Buleon A, Gallant DJ, Bouchet B, Mouille G, D'Hulst C, Kossmann J, et al. Starches from A to C (Chlamydomonas reinhardtii as a Model Microbial System to Investigate the Biosynthesis of the Plant Amylopectin Crystal). Plant Physiology. 1997;115(3):949-57.

3. Pfister B, Zeeman SC. Formation of starch in plant cells. Cellular and Molecular Life Sciences. 2016;73(14):2781-807.

4. D'Hulst C, Merida A. The priming of storage glucan synthesis from bacteria to plants: current knowledge and new developments. New Phytol. 2010;188(1):13-21.

5. Brust H, Orzechowski S, Fettke J. Starch and Glycogen Analyses. 2020.

6. Garz A, Sandmann M, Rading M, Ramm S, Menzel R, Steup M. Cell-to-Cell Diversity in a Synchronized Chlamydomonas Culture As Revealed by Single-Cell Analyses. Biophysical Journal. 2012;103(5):1078-86.

7. Buleon A, Cotte M, Putaux JL, d'Hulst C, Susini J. Tracking sulfur and phosphorus within single starch granules using synchrotron X-ray microfluorescence mapping. Biochim Biophys Acta. 2014;1840(1):113-9.

8. Wu AC, Morell MK, Gilbert RG. A Parameterized Model of Amylopectin Synthesis Provides Key Insights into the Synthesis of Granular Starch. PLOS ONE. 2013;8(6):e65768.

9. Vilaplana F, Gilbert RG. Analytical methodology for multidimensional size/branch-length distributions for branched glucose polymers using off-line 2-dimensional size-exclusion chromatography and enzymatic treatment. Journal of Chromatography A. 2011;1218(28):4434-44.

10. Rolland-Sabaté A, Colonna P, Mendez-Montealvo MG, Planchot V. Branching Features of Amylopectins and Glycogen Determined by Asymmetrical Flow Field Flow Fractionation Coupled with Multiangle Laser Light Scattering. Biomacromolecules. 2007;8(8):2520-32.

11. Broberg S, Koch K, Andersson R, Kenne L. A comparison between MALDI-TOF mass spectrometry and HPAEC-PAD analysis of debranched starch. Carbohydrate Polymers. 2000;43(3):2859.

12. Verbeke J, Penverne C, D’Hulst C, Rolando C, Szydlowski N. Rapid and sensitive quantification of C3- and C6-phosphoesters in starch by fluorescence-assisted capillary electrophoresis. Carbohydrate Polymers. 2016;152:784-91.

13. Helle S, Bray F, Putaux J-L, Verbeke J, Flament S, Rolando C, et al. Intra-Sample Heterogeneity of Potato Starch Reveals Fluctuation of Starch-Binding Proteins According to Granule Morphology. Plants. 2019;8(9):324.

14. Hizukuri S. Polymodal distribution of the chain lengths of amylopectins, and its significance. Carbohydrate Research. 1986;147(2):342-7. 